PROCEEDINGS OF THE

AMERICAN MATHEMATICAL SOCIETY

Volume 129, Number 5, Pages 1427-1435

S 0002-9939(00)05874-3

Article electronically published on October 25, 2000

\title{
INVARIANT PROJECTIONS AND CONVOLUTION OPERATORS
}

\author{
JACQUES DELAPORTE AND ANTOINE DERIGHETTI
}

(Communicated by Dale Alspach)

\begin{abstract}
We prove the existence of invariant projections $\mathcal{P}$ from the Banach space $P M_{p}(G)$ of $p$-pseudomeasures onto $P M_{p}(H)$ with $\operatorname{supp} \mathcal{P}(T) \subset \operatorname{supp} T$ for $H$ closed neutral subgroup of a locally compact group $G$. As a main application we obtain that every closed neutral subgroup is a set of $p$-synthesis in $G$ and in fact locally $p$-Ditkin in $G$. We also obtain an extension theorem concerning the Fourier algebra.
\end{abstract}

\section{INTRODUCTION}

For a locally compact group $G$, let $C V_{p}(G)$ be the Banach algebra of all convolution operators of $L^{p}(G)$ where $1<p<\infty$. In 1974 Lohoué [10] proved, for an amenable closed subgroup $H$ of $G$ (and $G \sigma$-compact), the existence of a projection of $C V_{p}(G)$ onto $C V_{p}(H)$. We obtain the existence of a projection $\mathcal{P}$ for the class of closed neutral subgroups. This class includes the following situations: (i) the normalizer of $H$ in $G$ is open in $G$; (ii) $G \in[S I N]_{H}$. We write $G \in[S I N]_{H}$ if there is a fundamental system of neighborhoods $U$ of $e$ in $G$ such that $h U h^{-1}=U$ for every $h \in H$. The class $[S I N]_{H}$ has been thoroughly investigated by Henrichs [5].

The existence of such a $\mathcal{P}$ for $H$ normal in $G$ is already in [1]. In fact we are now able to show that $\operatorname{supp} \mathcal{P}(T) \subset \operatorname{supp} T$ and $\mathcal{P}(u T)=\left(\operatorname{Res}_{H} u\right) \mathcal{P}(T)$ for $u \in A_{p}(G)$ and $T \in C V_{p}(G)$. The existence of a $\mathcal{P}$ with these properties is new even for $G$ abelian and $p \neq 2$; if $p=2$ and $G$ is abelian the result is due to C. Herz [6]. As a main application we prove that every closed neutral subgroup is a set of $p$-synthesis in $G$ and locally $p$-Ditkin in $G$. A closed subset $F$ of $G$ is locally $p$-Ditkin in $G$ (see [3], p. 102) if for every $\varepsilon>0$ and every $u \in A_{p}(G) \cap C_{00}(G)$ with $\operatorname{Res}_{F} u=0$ there is $v \in A_{p}(G) \cap C_{00}(G)$ with $\operatorname{supp} v \cap F=\emptyset$ and $\|u-u v\|_{A_{p}(G)}<\varepsilon$. $F$ is $p$-Ditkin in $G$ if for every $\varepsilon>0$ and every $u \in A_{p}(G)$ with $\operatorname{Res}_{F} u=0$ there is $v \in A_{p}(G) \cap C_{00}(G)$ with $\operatorname{supp} v \cap F=\emptyset$ and $\|u-u v\|_{A_{p}(G)}<\varepsilon$. The method used in the construction of $\mathcal{P}$ gives the following extension theorem concerning the Figà-Talamanca Herz algebra of $G$ : given $u \in A_{p}(H) \cap C_{00}(H), \varepsilon>0$, and an open subset $\Omega$ of $G$ with supp $u \subset \Omega$, there exists $v \in A_{p}(G) \cap C_{00}(G)$ with $\operatorname{Res}_{H} v=u$, $\|v\|_{A_{p}(G)} \leq\|u\|_{A_{p}(H)}+\varepsilon$ and $\operatorname{supp} v \subset \Omega$.

Received by the editors August 17, 1999.

1991 Mathematics Subject Classification. Primary 43A15, 43A07; Secondary 43A45, 43A46, 46J10.

Key words and phrases. Convolution operators, pseudomeasures, amenable groups, spectral synthesis, Ditkin sets, Fourier algebra, Figà-Talamanca Herz algebra.

This work was supported by the Swiss National Science Foundation. 
We have the pleasure to thank Professor Lohoué for stimulating conversations and Professor Roelke for the communication of unpublished results on neutral subgroups.

\section{DeFinitions AND PRELIMINARIES}

For the precise definitions of $C V_{p}(G), P M_{p}(G), A_{p}(G)$ and all the unexplained notations we refer to [1], p. 38. Let $H$ be an arbitrary closed subgroup of $G$ : for $T \in C V_{p}(H), i(T)$ denotes the image of $T$ under the inclusion $i$ of $H$ in $G$, as defined in [2], p. 76. We assume that $G / H$ admits a $G$-invariant measure. For $k, \ell \in C_{00}(G)$ the relation

$$
\left\langle\Lambda_{k, \ell}(T) \varphi, \psi\right\rangle_{L^{p}(H), L^{p^{\prime}}(H)}=\left\langle T \tau_{p}\left(k *_{H} \tau_{p} \varphi\right), \tau_{p^{\prime}}\left(\ell *_{H} \tau_{p^{\prime}} \psi\right)\right\rangle_{L^{p}(G), L^{p^{\prime}}(G)}
$$

defines a linear continuous map $\Lambda_{k, \ell}$ from $C V_{p}(G)$ into $C V_{p}(H)$. We have $\left\|\Lambda_{k, \ell}\right\|$ $\leq\left\|T_{H}|k|\right\|_{p}\left\|T_{H}|\ell|\right\|_{p^{\prime}}$ and $\Lambda_{k, \ell}\left(P M_{p}(G)\right) \subset P M_{p}(H)$ ([1], pp. 38-39).

The following unpublished result is due to Roelcke. Let $H$ be a closed neutral subgroup of $G(12)$. Then $e$ admits a fundamental system of neighborhoods $V$ with $H V=V H$. Indeed let $U$ be an open neighborhood of $e$ in $G$. There is an open $W$ with $e \in W, W=W^{-1}$ and $H W H \subset U H$. Then for $V=(H W H) \cap U$ one has precisely $H V=V H$.

A one-sided version of the following lemma is already in [2], p. 71.

Lemma 1. Let $G$ be a locally compact group, $H$ an arbitrary closed subgroup, $U$ a neighborhood of $e$ in $G$, and $W$ a neighborhood of $e$ in $H$. Then there exists $k \in C_{00}^{+}(G)$ with $\operatorname{supp} k \subset U,(\operatorname{supp} k) \cap H \subset W$ and

$$
\int_{H} k(h) d h=1, \int_{H} k(x h) d h \leq 1, \int_{H} \Delta_{H}\left(h^{-1}\right) k(h x) d h \leq 1
$$

for every $x \in G$.

Proof. There is an open neighborhood $U_{1}$ of $e$ in $G$ with $U_{1} \cap H \subset W$ and an open neighborhood $U_{2}$ of $e$ in $G$ with $U_{2}=U_{2}^{-1}$ and $U_{2} \subset U \cap U_{1}$. Let $K$ be a compact neighborhood of $e$ in $G$ with $K=K^{-1}$ and $K \subset U_{2}$. We choose $\varphi^{\prime} \in C_{00}^{+}(G)$ with $\varphi^{\prime}(K)=\{1\}$ and $\operatorname{supp} \varphi \subset U_{2}$. Consider also $\psi^{\prime} \in C_{00}^{+}(G / H)$ with $\psi^{\prime} \leq 1_{G / H}$, $\psi^{\prime}(H)=1$ and $\operatorname{supp} \psi^{\prime} \subset \omega(K)$. ( $\omega$ is the canonical map from $G$ to $G / H$.) Let

$$
\varphi=\frac{\varphi^{\prime}+\varphi^{\prime \vee}}{2}\left(\varphi^{\prime \vee}(x)=\varphi^{\prime}\left(x^{-1}\right)\right) \text { and } k^{\prime}(x)=\frac{\varphi(x) \psi^{\prime}(\omega(x))}{\int_{H} \varphi(x h) d h} \text { for } x \in A H,
$$

where $A=\{y \in G \mid \varphi(y)>0\}$ and $k^{\prime}(x)=0$ if $x \in G \backslash A H$. Taking into account that $G=A H \cup(G \backslash H K)$, we obtain that $k^{\prime} \in C_{00}^{+}(G), \operatorname{supp} k^{\prime} \subset \operatorname{supp} \varphi$ and $\int_{H} k^{\prime}(x h) d h \leq 1$ for every $x \in G$.

Now let $\omega^{\prime}$ be the canonical map from $G$ onto $H \backslash G$. We similarly choose $\psi^{\prime \prime} \in$ $C_{00}^{+}(H \backslash G)$ with $\psi^{\prime \prime} \leq 1_{H \backslash G}, \psi^{\prime \prime}(H)=1$, supp $\psi^{\prime \prime} \subset \omega^{\prime}(K)$ and

$$
k^{\prime \prime}(x)=\frac{\varphi(x) \psi^{\prime \prime}\left(\omega^{\prime}(x)\right)}{\int_{H} \Delta_{H}\left(h^{-1}\right) \varphi(h x) d h} \quad \text { for } x \in H A,
$$

$k^{\prime \prime}(x)=0$ for $x \in G \backslash H A$. We obtain that $k=\min \left\{k^{\prime}, k^{\prime \prime}\right\}$ satisfies all the required properties. 


\section{Projections of $C V_{p}(G)$ onto $C V_{p}(H)$}

According to Leischner and Roelcke [9] $H$ is said to be locally neutral in $G$ if there is a compact neighborhood $U_{0}$ of $e$ such that for every neighborhood $U$ of $e$ there is a neighborhood $V$ of $e$ with $(H V H) \cap U_{0} \subset U H$.

Proposition 2. Let $G$ be a locally compact group and $H$ a closed subgroup locally neutral in $G$. We assume that $G / H$ admits an invariant measure. Let $\left(r_{n}^{(j)}\right)_{n=1}^{\infty}$ be $m$ sequences of $L^{p}(H)$ and $\left(s_{n}^{(j)}\right)_{n=1}^{\infty}$ be $m$ sequences of $L^{p^{\prime}}(H)$. We assume that for every $1 \leq j \leq m, \sum_{n=1}^{\infty}\left\|r_{n}^{(j)}\right\|\left\|_{p}\right\| s_{n}^{(j)} \|_{p^{\prime}}<\infty$. Then, for every $\varepsilon>0$ and every open neighborhood $U$, there is $k, \ell \in C_{00}^{+}(G)$ with $\operatorname{supp} k, \operatorname{supp} \ell \subset U,\left\|\Lambda_{k, \ell}\right\| \leq 1$ and

$$
\sum_{n=1}^{\infty}\left|\left\langle\Lambda_{k, \ell}(i(S)) r_{n}^{(j)}, s_{n}^{(j)}\right\rangle_{L^{p}(H), L^{p^{\prime}}(H)}-\left\langle S r_{n}^{(j)}, s_{n}^{(j)}\right\rangle_{L^{p}(H), L^{p^{\prime}}(H)}\right| \leq \varepsilon|||S| \|_{p}
$$

for every $S \in C V_{p}(H)$ and every $1 \leq j \leq m$.

Proof. To avoid unessential technical difficulties, we suppose $m=1$ and $r_{n}^{(1)}, s_{n}^{(1)}$ $\in C_{00}(H)$ for every $n \in \mathbb{N}$. We put $r_{n}=r_{n}^{(1)}$ and $s_{n}=s_{n}^{(1)}$. There is $N \in$ $\mathbb{N}$ such that $\sum_{n=1+N}^{\infty}\left\|r_{n}\right\|_{p}\left\|s_{n}\right\|_{p^{\prime}}<\frac{\varepsilon_{1}}{8}$ where $0<\varepsilon_{1}<\min \{1, \varepsilon\}$. We can find a relatively compact neighborhood $V$ of $e$ in $H$ with $\left\|r_{n}-\left(r_{n}\right)_{h^{-1}} \Delta_{H}\left(h^{-1}\right)\right\|_{p}$, $\left\|s_{n}-\left(s_{n}\right)_{h^{-1}} \Delta_{H}\left(h^{-1}\right)\right\|_{p^{\prime}}<\frac{\varepsilon_{2}}{2}$ for every $h \in V$ and every $1 \leq n \leq N$. We have chosen $\varepsilon_{2}$ such that

$$
0<\varepsilon_{2}<\min \left\{\frac{\varepsilon_{1}}{2^{j+2}\left(1+\left\|r_{j}\right\|_{p}+\left\|s_{j}\right\|_{p^{\prime}}\right)} \mid 1 \leq j \leq N\right\} .
$$

Let $U_{0}$ be a compact neighborhood of $e$ in $G$ with $U_{0}=U_{0}^{-1}$ which guarantees the local neutrality of $H$ in $G$ : for every neighborhood $U^{\prime}$ of $e$ in $G$, there is a neighborhood $V^{\prime}$ of $e$ in $G$ with $U_{0} \cap\left(H V^{\prime} H\right) \subset U^{\prime} H$.

Let

$$
0<\varepsilon_{3}<\frac{\varepsilon_{1}}{40\left(1+\sum_{n=1}^{\infty}\left\|r_{n}\right\|_{p}\left\|s_{n}\right\|_{p^{\prime}}\right)} .
$$

There is a compact neighborhood $U_{1}$ of $e$ in $G$ with $\Delta_{G}(x)<1+\varepsilon_{3}$ for every $x \in U_{1}$. We choose an open neighborhood $U_{2}$ of $e$ in $G$ with $U_{2}^{2} \subset U_{1}$. According to Lemma 1, there is $k^{\prime} \in C_{00}^{+}(G)$ with $\int_{H} k^{\prime}(h) d h=1, \int_{H} k^{\prime}(h x) \Delta_{H}\left(h^{-1}\right) d h \leq 1$ for every $x \in G$, supp $k^{\prime} \subset U_{0} \cap U_{2} \cap U^{-1}$ and supp $k^{\prime} \cap H \subset V$. This implies, for every $1 \leq n \leq N,\left\|r_{n}-\operatorname{Res}_{H}\left(r_{n} *_{H} k^{\prime}\right)\right\|_{p} \leq \frac{\varepsilon_{2}}{2}$ and $\left\|s_{n}-\operatorname{Res}_{H}\left(s_{n} *_{H} k^{\prime}\right)\right\|_{p^{\prime}} \leq \frac{\varepsilon_{2}}{2}$. There is a relatively compact open neighborhood $U_{3}$ of $e$ in $G$ such that, for every $x \in U_{3}$ and every $1 \leq n \leq N,\left\|\left(r_{n} *_{H} k^{\prime}\right)_{x, H}-\operatorname{Res}_{H}\left(r_{n} *_{H} k^{\prime}\right)\right\|_{p}<\frac{\varepsilon_{2}}{2}$ and $\left\|\left(s_{n} *_{H} k^{\prime}\right)_{x, H}-\operatorname{Res}_{H}\left(s_{n} *_{H} k^{\prime}\right)\right\|_{p^{\prime}}<\frac{\varepsilon_{2}}{2}$. (We recall that, for a function $f$ on $G$ and for $x \in G, f_{x, H}$ denotes the function defined on $H$ by $f_{x, H}(h)=f(x h)$.) 
Now let $A$ be an arbitrary open neighborhood of $e$ in $G$ with $A \subset U_{3}$. Then, for every $S \in C V_{p}(H)$ and every $1 \leq n \leq N$, we have

$$
\begin{array}{r}
\frac{\left\langle i(S) 1_{A H}\left(r_{n} *_{H} k^{\prime}\right), 1_{A H}\left(s_{n} *_{H} k^{\prime}\right)\right\rangle_{L^{p}(G), L^{p^{\prime}}(G)}}{\dot{m}(\omega(A))}-\left\langle S r_{n}, s_{n}\right\rangle_{L^{p}(H), L^{p^{\prime}}(H)} \\
=\frac{1}{\dot{m}(\omega(A))} \int_{G} 1_{A H}(x) \beta(x)\left(\left\langle S\left(r_{n} *_{H} k^{\prime}\right)_{x, H},\left(s_{n} *_{H} k^{\prime}\right)_{x, H}\right\rangle_{L^{p}(H), L^{p^{\prime}}(H)}\right. \\
\left.-\left\langle S r_{n}, s_{n}\right\rangle_{L^{p}(H), L^{p^{\prime}}(H)}\right) d x
\end{array}
$$

where $\dot{m}(\omega(A))=\int_{\omega(A)} d \dot{x}$ and $\beta$ is a Bruhat function for $H, G$.

Taking into account that we have, for $x=u h$ with $u \in A, h \in H$,

$$
\begin{aligned}
& \left\langle S\left(r_{n} *_{H} k^{\prime}\right)_{x, H},\left(s_{n} *_{H} k^{\prime}\right)_{x, H}\right\rangle_{L^{p}(H), L^{p^{\prime}}(H)} \\
& \quad=\left\langle S\left(r_{n} *_{H} k^{\prime}\right)_{u, H},\left(s_{n} *_{H} k^{\prime}\right)_{u, H}\right\rangle_{L^{p}(H), L^{p^{\prime}}(H)},
\end{aligned}
$$

we obtain

$$
\begin{aligned}
& \mid \frac{\left\langle i(S) 1_{A H}\left(r_{n} *_{H} k^{\prime}\right), 1_{A H}\left(s_{n} *_{H} k^{\prime}\right)\right\rangle_{L^{p}(G), L^{p^{\prime}}(G)}}{\dot{m}(\omega(A))}-\left\langle S r_{n}, s_{n}\right\rangle_{L^{p}(H), L^{p^{\prime}}(H)} \\
& \leq\||| S \mid\|_{p} \varepsilon_{2}\left(1+\left\|r_{n}\right\|_{p}+\left\|s_{n}\right\|_{p^{\prime}}\right)
\end{aligned}
$$

for every $1 \leq n \leq N$.

Let $K$ be a compact subset of $H$ with $e \in K$ and supp $r_{n} \cup \operatorname{supp} s_{n} \subset K$ for every $1 \leq n \leq N$. There is an open neighborhood $U_{4}$ of $e$ in $G$ such that $U_{4}^{-1}=U_{4}$ and $U_{4} \subset U_{2} \cap U_{3}$. There is an open neighborhood $U_{5}$ of $e$ in $G$ such that $k U_{5} k^{-1} \subset U_{4}$ for every $k \in K$. By assumption we can find an open neighborhood $U_{6}$ of $e$ in $G$ such that $\left(H U_{6} H\right) \cap U_{0} \subset U_{5} H$. This implies $\left(H U_{6} H\right) \cap K U_{0} H \subset U_{4} H$. Consider $U_{8}=\left(H U_{7} H\right) \cap U_{4}$ where $U_{7}$ is an open neighborhood of $e$ in $G$ with $U_{7}^{-1}=U_{7}$ and $U_{7} \subset U_{6}$. Roelcke's argument gives $\left(K U_{0} K^{-1}\right) \cap U_{8} H=\left(K U_{0} K^{-1}\right) \cap H U_{8}$. If we take into account that $\operatorname{supp}\left(r_{n} *_{H} k^{\prime}\right), \operatorname{supp}\left(s_{n} *_{H} k^{\prime}\right) \subset K U_{0} K^{-1}(1 \leq n \leq N)$, we obtain, for $1 \leq n \leq N$,

$$
1_{U_{8} H}\left(r_{n} *_{H} k^{\prime}\right)=1_{H U_{8}}\left(r_{n} *_{H} k^{\prime}\right) \text { and } 1_{U_{8} H}\left(s_{n} *_{H} k^{\prime}\right)=1_{H U_{8}}\left(s_{n} *_{H} k^{\prime}\right)
$$

Let

$$
k^{\prime \prime}=\frac{\tau_{p}\left(1_{H U_{8}} k^{\prime}\right)}{\dot{m}\left(\omega\left(U_{8}\right)\right)^{1 / p}} \quad \text { and } \quad \ell^{\prime \prime}=\frac{\tau_{p^{\prime}}\left(1_{H U_{8}} k^{\prime}\right)}{\dot{m}\left(\omega\left(U_{8}\right)\right)^{1 / p^{\prime}}} .
$$

Then, for $1 \leq n \leq N$

$$
\begin{aligned}
& \left\langle\Lambda_{k^{\prime \prime}, \ell^{\prime \prime}}(i(S)) r_{n}, s_{n}\right\rangle_{L^{p}(H), L^{p^{\prime}}(H)} \\
& =\frac{\left\langle i(S) 1_{U_{8} H}\left(r_{n} *_{H} k^{\prime}\right), 1_{U_{8} H}\left(s_{n} *_{H} k^{\prime}\right)\right\rangle_{L^{p}(G), L^{p^{\prime}}(G)}}{\dot{m}\left(\omega\left(U_{8}\right)\right)} .
\end{aligned}
$$


From the inequality

$$
\begin{aligned}
& \sum_{n=1}^{\infty} \mid\left\langle S r_{n}, s_{n}\right\rangle_{L^{p}(H), L^{p^{\prime}(H)}}-\left\langle\Lambda_{k^{\prime \prime}, \ell^{\prime \prime}}(i(S)) r_{n}, s_{n}\right\rangle_{L^{p}(H), L^{p^{\prime}(H)}} \mid \\
& \leq \sum_{n=1}^{N}\left|\left\|S\left|\|_{p} \varepsilon_{2}\left(1+\left\|r_{n}\right\|_{p}+\left\|s_{n}\right\|_{p^{\prime}}\right)+\sum_{n=N+1}^{\infty}\right|\left\langle S r_{n}, s_{n}\right\rangle_{L^{p}(H), L^{p^{\prime}(H)} \mid} \mid\right.\right. \\
&+\sum_{n=N+1}^{\infty}\left|\left\langle\Lambda_{k^{\prime \prime}, \ell^{\prime \prime}}(i(S)) r_{n}, s_{n}\right\rangle_{L^{p}(H), L^{p^{\prime}}(H)}\right|,
\end{aligned}
$$

we get

$$
\begin{gathered}
\sum_{n=1}^{\infty}\left|\left\langle S r_{n}, s_{n}\right\rangle_{L^{p}(H), L^{p^{\prime}}(H)}-\left\langle\Lambda_{k^{\prime \prime}, \ell^{\prime \prime}}(i(S)) r_{n}, s_{n}\right\rangle_{L^{p}(H), L^{p^{\prime}}(H)}\right| \\
\leq \frac{3 \varepsilon_{1}\||| S \mid\|_{p}}{8}+\frac{\varepsilon_{1}\left\|\left.|| S\left|\left\|\left.\right|_{p}\right\| T_{H} k^{\prime \prime}\right|\right|_{p}\right\| T_{H} \ell^{\prime \prime} \|_{p^{\prime}}}{8} .
\end{gathered}
$$

To estimate $\left\|T_{H} k^{\prime \prime}\right\|_{p}\left\|T_{H} \ell^{\prime \prime}\right\|_{p^{\prime}}$, observe that, for $x=u h^{\prime}$ with $u \in U_{8}, h^{\prime} \in H$, $\int_{H}\left(\tau_{p} k^{\prime}\right)(x h) d h=\int_{H} k^{\prime}\left(h u^{-1}\right) \Delta_{G}\left(h u^{-1}\right)^{1 / p} \Delta_{H}\left(h^{-1}\right) d h$. But $u^{-1} \in U_{1}$ implies

$$
\int_{H}\left(\tau_{p} k^{\prime}\right)(x h) d h \leq\left(1+\varepsilon_{3}\right)^{1 / p} \int_{H} k^{\prime}\left(h u^{-1}\right) \Delta_{G}(h)^{1 / p} \Delta_{H}\left(h^{-1}\right) d h .
$$

From $\int_{H} k^{\prime}\left(h u^{-1}\right) \Delta_{G}(h)^{1 / p} \Delta_{H}\left(h^{-1}\right) d h=\int_{U_{1} \cap H} k^{\prime}\left(h u^{-1}\right) \Delta_{G}(h)^{1 / p} \Delta_{H}\left(h^{-1}\right) d h$, we therefore get $\left\|T_{H} k^{\prime \prime}\right\|_{p} \leq\left(1+\varepsilon_{3}\right)^{2 / p}$ and similarly $\left\|T_{H} \ell^{\prime \prime}\right\|_{p^{\prime}} \leq\left(1+\varepsilon_{3}\right)^{2 / p^{\prime}}$. This gives, for every $S \in C V_{p}(H)$,

$$
\sum_{n=1}^{\infty}\left|\left\langle S r_{n}, s_{n}\right\rangle_{L^{p}(H), L^{p^{\prime}}(H)}-\left\langle\Lambda_{k^{\prime \prime}, \ell^{\prime \prime}}(i(S)) r_{n}, s_{n}\right\rangle_{L^{p}(H), L^{p^{\prime}(H)}}\right| \leq \frac{7 \varepsilon_{1}}{8}\||| S \mid\|_{p} .
$$

Consider now $f, g \in C_{00}^{+}(G / H)$ with $\left\|f-\frac{1_{\omega\left(U_{8}\right)}}{\dot{m}\left(\omega\left(U_{8}\right)\right)^{1 / p}}\right\|\left\|_{p},\right\| g-\frac{1_{\omega\left(U_{8}\right)}}{\dot{m}\left(\omega\left(U_{8}\right)\right)^{1 / p^{\prime}}}\|\|_{p^{\prime}}$ both smaller than $\frac{\varepsilon_{4}}{\left(1+\left\|T_{H} \tau_{p} k^{\prime}\right\|_{\infty}\right)\left(1+\left\|T_{H} \tau_{p^{\prime}} k^{\prime}\right\|_{\infty}\right)}$, where

$$
0<\varepsilon_{4}<\frac{\varepsilon_{3}}{\left(1+2^{2 / p}+2^{2 / p^{\prime}}\right)\left(1+\sum_{n=1}^{\infty}\left\|r_{n}\right\|_{p}\left\|s_{n}\right\|_{p^{\prime}}\right)}
$$

Putting $k^{\prime \prime \prime}=(f \circ \omega) \tau_{p} k^{\prime}$ and $\ell^{\prime \prime \prime}=(g \circ \omega) \tau_{p^{\prime}} k^{\prime}$, we have successively

$$
\left\|T_{H}\left(\left|k^{\prime \prime \prime}-k^{\prime \prime}\right|\right)\right\|_{p},\left\|T_{H}\left(\left|\ell^{\prime \prime \prime}-\ell^{\prime \prime}\right|\right)\right\|_{p^{\prime}}<\varepsilon_{4}
$$

$\left\|T_{H} k^{\prime \prime \prime}\right\|_{p}<\varepsilon_{4}+\left(1+\varepsilon_{3}\right)^{2 / p}$ and $\left\|T_{H} \ell^{\prime \prime \prime}\right\|_{p^{\prime}}<\varepsilon_{4}+\left(1+\varepsilon_{3}\right)^{2 / p^{\prime}}$. Finally it suffices to choose $k=\frac{k^{\prime \prime \prime}}{\varepsilon_{4}+\left(1+\varepsilon_{3}\right)^{2 / p}}$ and $\ell=\frac{\ell^{\prime \prime \prime}}{\varepsilon_{4}+\left(1+\varepsilon_{3}\right)^{2 / p^{\prime}}}$ to obtain

$$
\sum_{n=1}^{\infty}\left|\left\langle S r_{n}, s_{n}\right\rangle_{L^{p}(H), L^{p^{\prime}(H)}}-\left\langle\Lambda_{k, \ell}(i(S)) r_{n}, s_{n}\right\rangle_{L^{p}(H), L^{p^{\prime}(H)}}\right| \leq \varepsilon\||| S \mid\| \|_{p}
$$

with $\left\|\Lambda_{k, \ell}\right\| \leq 1$ and $\operatorname{supp} k, \operatorname{supp} \ell \subset U$. 
We are now ready to prove our first result concerning projections of $C V_{p}(G)$ onto $C V_{p}(H)$.

Theorem 3. Let $G$ be a locally compact group and $H$ a closed subgroup, locally neutral in $G$. We assume that $G / H$ admits an invariant measure. Then there is a linear contraction $Q$ from $\mathcal{L}\left(L^{p}(G)\right.$ ) (the Banach space of all bounded operators of $\left.L^{p}(G)\right)$ into $\mathcal{L}\left(L^{p}(H)\right)$ such that:

(1) $Q(T) \in C V_{p}(H)$ for every $T \in C V_{p}(G)$,

(2) $\operatorname{supp} Q(T) \subset \operatorname{supp} T$ for every $T \in C V_{p}(G)$,

(3) $Q(i(S))=S$ for every $S \in C V_{p}(H)$,

(4) $Q(T) \in P M_{p}(H)$ for every $T \in P M_{p}(G)$.

Proof. Let $\mathcal{A}$ be the set of all pairs $\left(\left(r_{n}\right)_{n=1}^{\infty},\left(s_{n}\right)_{n=1}^{\infty}\right)$ where $\left(r_{n}\right)_{n=1}^{\infty}$ is a sequence of $L^{p}(H)$ and $\left(s_{n}\right)_{n=1}^{\infty}$ is a sequence of $L^{p^{\prime}}(H)$ with $\sum_{n=1}^{\infty}\left\|r_{n}\right\|\left\|_{p}\right\| s_{n} \|_{p^{\prime}}<\infty$. We denote by $\mathcal{E}$ the set of all maps $F$ from $\mathcal{L}\left(L^{p}(G)\right) \times L^{p}(H) \times L^{p^{\prime}}(H)$ to $\mathbb{C}$, linear in the first two variables, conjugate linear in the third one, and for which there is a positive real number $C$ with $|F(T, \varphi, \psi)| \leq C\left|\|T \mid\|_{p}\|\varphi\|_{p}\|\psi\|_{p^{\prime}}\right.$. For $F \in \mathcal{E}$ we put $\|F\|=\sup \left\{|F(T, \varphi, \psi)| \mid\|T\|_{p} \leq 1,\|\varphi\|_{p} \leq 1,\|\psi\|_{p^{\prime}} \leq 1\right\}$. For $k, \ell \in$ $C_{00}(G), F_{k, \ell}(T, \varphi, \psi)=\left\langle\Lambda_{k, \ell}(T) \varphi, \psi\right\rangle_{L^{p}(H), L^{p^{\prime}}(H)}$ is an element of $\mathcal{E}$ with $\left\|F_{k, \ell}\right\| \leq$ $\left\|T_{H}|k|\right\|_{p}\left\|T_{H}|\ell|\right\|_{p^{\prime}}$

Let $A$ be a finite subset of $\mathcal{A}, B$ a finite subset of $C V_{p}(H), U$ an open neighborhood of $e$ in $G$ and $\varepsilon>0$. Proposition 2 implies precisely that the set

$$
\begin{aligned}
K_{A, B, U, \varepsilon}= & \left\{F_{k, \ell}\left|k, \ell \in C_{00}^{+}(G) \quad \| F_{k, \ell}\right| \mid \leq 1, \operatorname{supp} k, \text { supp } \ell \subset U,\right. \\
& \sum_{n=1}^{\infty}\left|F_{k, \ell}\left(i(S), r_{n}, s_{n}\right)-\left\langle S r_{n}, s_{n}\right\rangle_{L^{p}(H), L^{p^{\prime}}(H)}\right|<\varepsilon \\
& \text { for every } \left.\left(\left(r_{n}\right)_{n=1}^{\infty},\left(s_{n}\right)_{n=1}^{\infty}\right) \in A \text { and every } S \in B\right\}
\end{aligned}
$$

is nonempty. Let $\bar{K}_{A, B, U, \varepsilon}$ be the closure of $K_{A, B, U, \varepsilon}$ with respect to the topology $\sigma\left(\mathcal{E}, \mathcal{L}\left(L^{p}(G)\right) \times L^{p}(H) \times L^{p^{\prime}}(H)\right)$. The set $\cap\left\{\bar{K}_{A, B, U, \varepsilon} \mid A\right.$ finite subset of $\mathcal{A}$, $B$ finite subset of $C V_{p}(H), 0<\varepsilon<1, U$ open neighborhood of $e$ in $G$ \} is not empty. Choose $J$ in this set. There is a linear map $Q$ from $\mathcal{L}\left(L^{p}(G)\right)$ to $\mathcal{L}\left(L^{p}(H)\right)$ with $J(T, \varphi, \psi)=\langle Q(T) \varphi, \psi\rangle_{L^{p}(H), L^{p^{\prime}}{ }_{(H)}}$ for $T \in \mathcal{L}\left(L^{p}(G)\right), \varphi \in L^{p}(H), \psi \in$ $L^{p^{\prime}}(H)$. Clearly $Q$ satisfies conditions (1) to (4).

Let $H$ be a closed subgroup of $G$ for which there is a linear map $Q$ from $C V_{p}(G)$ onto $C V_{p}(H)$ satisfying conditions (3) and (4) of Theorem 3. Then $H$ is a set of $p$-synthesis in $G$. Indeed let $T \in P M_{p}(G)$ with supp $T \subset H$ and $u \in A_{p}(G)$ with $\operatorname{Res}_{H} u=0$. According to Lohoué ([11, Théorème 5, p. 190), there is an $S \in$ $C V_{p}(H)$ with $i(S)=T$. From $Q(i(S)) \in P M_{p}(H)$ we deduce that $S \in P M_{p}(H)$ and therefore $\langle u, T\rangle_{A_{p}(G), P M_{p}(G)}=\left\langle\operatorname{Res}_{H} u, S\right\rangle_{A_{p}(H), P M_{p}(H)}=0$.

Corollary 4. Let $G$ be a locally compact group and $H$ a closed subgroup, locally neutral in $G$, for which $G / H$ admits an invariant measure. Then $H$ is a set of p-synthesis of $G$.

The following extension theorem was proved by C. Herz for $G$ second countable and $H$ normal in $G([7]$, p. 115). 
Corollary 5. Let $G$ be a locally compact group and $H$ a closed subgroup as in Theorem 3. Given $u \in A_{p}(H) \cap C_{00}(H), \varepsilon>0$ and an open subset $\Omega$ of $G$ with $\operatorname{supp} u \subset$ $\Omega$, there exists $v \in A_{p}(G) \cap C_{00}(G)$ with $\operatorname{Res}_{H} v=u,\|v\|_{A_{p}(G)} \leq\|u\|_{A_{p}(H)}+\varepsilon$ and $\operatorname{supp} v \subset \Omega$.

Proof. According to [7], p. 115, it suffices to find $v \in A_{p}(G) \cap C_{00}(G)$ with supp $v \subset$ $\Omega,\|v\|_{A_{p}(G)} \leq\|u\|_{A_{p}(H)}$ and $\left\|u-\operatorname{Res}_{H} v\right\|_{A_{p}(H)}<\varepsilon$.

There is $\left(r_{n}\right)_{n=1}^{\infty}$ and $\left(s_{n}\right)_{n=1}^{\infty}$, two sequences of $C_{00}(H)$, such that $u=\sum_{n=1}^{\infty} \bar{r}_{n} * \check{s}_{n}$ and $\sum_{n=1}^{\infty}\left\|r_{n}\right\|_{L^{p}(H)}\left\|s_{n}\right\|_{L^{p^{\prime}(H)}}<\infty$. There also exists an open neighborhood $U$ of $e$ in $G$ such that $U \operatorname{supp} u U^{-1} \subset \Omega$. There is $k, \ell \in C_{00}^{+}(G)$ with $\operatorname{supp} k, \operatorname{supp} \ell \subset U$, $\left\|\Lambda_{k, \ell}\right\| \leq 1$ and

$$
\sum_{n=1}^{\infty}\left|\left\langle\Lambda_{k, \ell}(i(S)) \tau_{p} r_{n}, \tau_{p^{\prime}} s_{n}\right\rangle_{L^{p}(G), L^{p^{\prime}}(G)}-\left\langle S \tau_{p} r_{n}, \tau_{p^{\prime}} s_{n}\right\rangle_{L^{p}(H), L^{p^{\prime}(H)}}\right| \leq \varepsilon\||| S \mid\|_{p}
$$

for every $S \in C V_{p}(H)$. There exists a unique $v \in A_{p}(G)$ such that

$$
\langle v, i(S)\rangle_{A_{p}(G), P M_{p}(G)}=\left\langle u, \Lambda_{k, l}(S)\right\rangle_{A_{p}(H), P M_{p}(H)}
$$

for every $S \in P M_{p}(H)$. From

$$
\begin{gathered}
\left|\langle u, S\rangle_{A_{p}(H), P M_{p}(H)}-\left\langle\operatorname{Res}_{H} v, S\right\rangle_{A_{p}(H), P M_{p}(H)}\right| \\
\leq \sum_{n=1}^{\infty}\left|\left\langle S \tau_{p} r_{n}, \tau_{p^{\prime}} s_{n}\right\rangle_{L^{p}(H), L^{p^{\prime}(H)}}-\left\langle\Lambda_{k, l}(i(S)) \tau_{p} r_{n}, \tau_{p^{\prime}} s_{n}\right\rangle_{L^{p}(G), L^{p^{\prime}}(G)}\right| \leq \varepsilon \||| S||_{p}
\end{gathered}
$$

we get $\left\|u-\operatorname{Res}_{H} v\right\|_{A_{p}(H)} \leq \varepsilon$ with $\operatorname{supp} v \subset \operatorname{supp} k \operatorname{supp} u(\operatorname{supp} \ell)^{-1}$.

Remark. Suppose $G$ is abelian. According to J. Inoue [8] for every neighborhood $U$ of $e$ in $G$ there is a linear isometric map $\Omega$ of $A_{2}(H)$ into $A_{2}(G)$ with $\operatorname{Res}_{H} \circ \Omega=$ $\operatorname{id}_{A_{2}(H)}$ (such a map is called a linear lifting) and $\operatorname{supp} \Omega(u) \subset(\operatorname{supp} u) U$. By duality we easily derive the existence of a projection of $P M_{2}(G)$ onto $P M_{2}(H)$ as in Theorem 3. On the other hand B. Forrest [4] has shown that for $G$ amenable and $H$ closed abelian normal subgroup of $G$ a linear lifting does not always exist. Consequently the map $Q$ of Theorem 3 can be considered as a substitute, for $G$ nonabelian, to the nonexistence of linear liftings of $A_{2}(H)$ into $A_{2}(G)$.

\section{INVARIANT PROJECTIONS}

In [1] we proved for $H$ normal in $G$ the existence of a projection $\mathcal{P}$ of $C V_{p}(G)$ onto $C V_{p}(H)$ satisfying the condition $\mathcal{P}(u T)=\left(\operatorname{Res}_{H} u\right) \mathcal{P}(T)$; howewer condition (2) of Theorem 3 was out of our reach. A projection $\mathcal{P}$ with $\mathcal{P}(u T)=\left(\operatorname{Res}_{H} u\right) \mathcal{P}(T)$ will be called an invariant projection.

Theorem 6. Let $G$ be a locally compact group and $H$ a closed neutral subgroup of $G$. There is an invariant projection $\mathcal{P}$ of $C V_{p}(G)$ onto $C V_{P}(H)$ which also satisfies all conditions of Theorem 3.

Proof. Let $X$ be the set of all maps $f$ from $\mathcal{L}\left(L^{p}(G)\right) \times L^{p}(G) \times L^{p^{\prime}}(G)$ to $\mathbb{C}$ which are linear in the first two variables and conjugate linear in the third one and for which there is a positive real number $C$ with $|f(T, \varphi, \psi)| \leq C \mid\|T\|\left\|_{p}\right\| \varphi\left\|_{p}\right\| \psi \|_{p^{\prime}}$. 
For $a, b \in S(G / H)(S(G / H)$ is the set of all bounded mesurable functions on $G / H$ with compact support), $T \in \mathcal{L}\left(L^{p}(G)\right), \varphi, \psi \in C_{00}(G)$ we define

$$
g_{a, b}(T, \varphi, \psi)=\int_{G / H}\langle T B(\varphi, a)(\dot{t}), B(\psi, b)(\dot{t})\rangle_{L^{p}(G), L^{p^{\prime}}(G)} d \dot{t}
$$

with $B(\varphi, a)(\dot{t})(x)=\varphi(x) a\left(x^{-1} \dot{t}\right)$ for $x, t \in G$. Let $K$ be a compact subset of $G$ with $K \cap H=\emptyset$ and $\varepsilon>0$. At first we show that the set $D_{K, \varepsilon}=\left\{g_{a, b} \mid a, b \in\right.$ $S(G / H), a, b \geq 0,\|a\|_{p}\|b\|_{p^{\prime}}<1+\varepsilon$, there is an open neighborhood $U$ of $e$ in $G$ with $\operatorname{Res}_{H U} v=1, \operatorname{supp} v \cap K U=\emptyset$ where $\left.v(x)=\int_{G / H} a(x \dot{y}) b(\dot{y}) d \dot{y}\right\}$ is nonempty. We indeed choose an open neighborhood $U_{1}$ of $e$ in $G$ with $U_{1}=U_{1}^{-1}$ and $K U_{1} \cap H U_{1}^{2}=$ $\emptyset$. There is an open neighborhood $U_{2}$ of $e$ in $G$ with $U_{2}^{-1}=U_{2}, U_{2} \subset U_{1}$ and $U_{2} H=H U_{2}$. Let $U_{3}$ be an open neighborhood of $e$ in $G$ relatively compact with $\bar{U}_{3} \subset U_{2}$ and $U_{3} H=H U_{3}$. This implies that $\bar{U}_{3} H=H \bar{U}_{3}$. There is an open subset $\dot{U}_{4}$ of $G / H$ with $\dot{U}_{4} \supset \omega\left(\bar{U}_{3}\right)$ and $\dot{m}\left(\dot{U}_{4}-\omega\left(\bar{U}_{3}\right)\right)<\left((1+\varepsilon)^{p^{\prime}}-1\right) \dot{m}\left(\omega\left(\bar{U}_{3}\right)\right)$. Let $U_{5}=\omega^{-1}\left(\dot{U}_{4}\right) \cap U_{2}$. We consider an open neighborhood $U_{6}$ of $e$ with $U_{6}^{-1}=U_{6}$ and $U_{6} \bar{U}_{3} \subset U_{5}$. Let $v(x)=\int_{G / H} a(x \dot{y}) b(\dot{y}) d \dot{y}$ with $a=\frac{1_{\omega\left(\bar{U}_{3}\right)}}{\dot{m}\left(\omega\left(\bar{U}_{3}\right)\right)}$ and $b=1_{\omega\left(U_{6} \bar{U}_{3}\right)}$. We have

$$
\|a\|_{p}\|b\|_{p^{\prime}} \leq\left(\frac{\dot{m}\left(\omega\left(U_{5}\right)\right)}{\dot{m}\left(\omega\left(\bar{U}_{3}\right)\right)}\right)^{\frac{1}{p^{\prime}}}<1+\varepsilon .
$$

Suppose $v(x) \neq 0$; there is $\dot{y} \in G / H$ with $x \dot{y} \in \omega\left(\bar{U}_{3}\right)$ and $\dot{y} \in \omega\left(U_{6} \bar{U}_{3}\right)$. This implies $x \in \bar{U}_{3} H U_{5}^{-1}$ and consequently $x \in H U_{1}^{2}$. Let $x \in H U_{6}$ for every $\dot{y} \in \omega\left(\bar{U}_{3}\right)$ $x^{-1} \dot{y} \in \omega\left(U_{6} H \bar{U}_{3}\right)$ but $\omega\left(U_{6} H \bar{U}_{3}\right)=\omega\left(U_{6} \bar{U}_{3}\right)$; this implies precisely $v(x)=1$. Let $g$ be an element of $\cap\left\{\bar{D}_{K, \varepsilon} \mid K\right.$ compact subset of $G$ with $K \cap H=\emptyset, 0<$ $\varepsilon<1\}$ where $\bar{D}_{K, \varepsilon}$ is the closure of $D_{K, \varepsilon}$ in $X$ with respect to the topology $\sigma\left(X, \mathcal{L}\left(L^{p}(G)\right) \times L^{p}(G) \times L^{p^{\prime}}(G)\right)$. Let $P$ be the corresponding map of $\mathcal{L}\left(L^{p}(G)\right)$ to itself. It suffices to consider $Q \circ P$ where $Q$ is the map of Theorem 3 .

Corollary 7. Let $G$ be a locally compact group, $H$ a closed neutral subgroup of $G$ and $F$ a closed subset of $H$. If $F$ is locally $p$-Ditkin $(1<p<\infty)$ with respect to $H$, then $F$ is locally $p$-Ditkin with respect to $G$.

Proof. Let $u \in A_{p}(G), T \in C V_{p}(G)$ with $\operatorname{Res}_{F} u=0$ and $\operatorname{supp}(u T) \subset F$. It suffices to show that $u T=0$. There is $S \in C V_{p}(H)$ such that $u T=i(S)$. Let $\mathcal{P}$ be the invariant projection of Theorem 6. From supp $\mathcal{P}(u T) \subset \operatorname{supp} u T$ and $\mathcal{P}(u T)=\left(\operatorname{Res}_{H} u\right) \mathcal{P}(T)=S$ we deduce that $S=0$ and therefore $u T=0$.

Remark. For $H$ normal in $G$, this corollary is already in [3], p. 103. Our proof there was completely different: it was based on the use of $A_{p}(G / H)$. The present approach is not only more conceptual but permits us to treat the case of certain interesting nonnormal subgroups: $H$ compact or $G \in[S I N]_{H}$.

\section{REFERENCES}

1. J. Delaporte and A. Derighetti, p-pseudomeasures and closed subgroups, Mh. Math. 119 (1995), 37-47. MR 97a:43001

2. A. Derighetti, Relations entre les convoluteurs d'un groupe localement compact et ceux d'un sous-groupe fermé, Bull. Sc. math. $2^{\circ}$ Série, 106 (1982), 69-84. MR 83j:43008 
3. A. Derighetti, Quelques observations concernant les ensembles de Ditkin d'un groupe localement compact, Mh. Math. 101 (1986), 95-113. MR 88c:43006

4. B. Forrest, Complemented ideals in the Fourier algebra and the Radon Nikodym property, Trans. Amer. Math. Soc. 333 (1992), 689-700. |MR 92m:43003

5. R. W. Henrichs, Ueber Fortsetzung positiv definiter Funktionen, Math. Ann. 232 (1978), 131-150. MR 58:2022

6. C. S. Herz, The spectral theory of bounded functions, Trans. Amer. Math. 94 (1960), 181-232. MR 24:A1627

7. MR 50:7956

8. J. Inoue, On a lifting problem of Fourier-Stieltjes transforms of measures, Hokkaido Math. J. 12 (1983), 112-118. MR 84f:43004

9. M. Leischner and W. Roelcke, On neutral subgroups of topological groups, Math. Ann. 282 (1988), 113-129. Math. 266. MR 90c:22005

10. N. Lohoué, Sur les convoluteurs de $L^{p}(G)$, C.R. Acad. Sc. Paris 278 (1974), 1543-1545. MR 49:11151

11. Estimations $L^{p}$ des coefficients de représentation et opérateurs de convolution, Adv. Math. 38 (1980), 178-221. MR 82m:43004

12. W. Roelcke and S. Dierolf, Uniform structures on topological groups and their quotients, McGraw-Hill, New York, 1981. MR 83j:22001

Institut de Mathématiques, Faculté des Sciences, Université de Lausanne, CH-1015 LAUSANNE-DORIGNY, SWITZERLAND

E-mail address: jdelaporte@mail.vtx.ch

Institut de Mathématiques, Faculté des Sciences, Université de Lausanne, CH-1015 LAUSANNE-DorignY, Switzerland

E-mail address: antoine.derighetti@ima.unil.ch 